\title{
The Abel-Zeilberger Algorithm
}

\author{
William Y.C. Chen ${ }^{1} \quad$ Qing-Hu Hou ${ }^{2} \quad$ Hai-Tao Jin ${ }^{3}$ \\ Center for Combinatorics, LPMC-TJKLC \\ Nankai University, Tianjin 300071, P.R. China \\ ${ }^{1}$ chen@nankai.edu.cn, ${ }^{2}$ hou@nankai.edu.cn, ${ }^{3}$ jinht1006@mail.nankai.edu.cn
}

Submitted: May 1, 2011; Accepted: Jul 31, 2011; Published: Aug 12, 2011

Mathematics Subject Classification: 33F10, 05A19, 39A10

Dedicated to Professor Doron Zeilberger on the occasion of his 60th birthday

\begin{abstract}
By combining Abel's lemma on summation by parts with Zeilberger's algorithm, we give an algorithm, called the Abel-Zeilberger algorithm, to find recurrence relations for definite summations. The role of Abel's lemma can be extended to the case of linear difference operators with polynomial coefficients. This approach can be used to verify and discover identities involving harmonic numbers and derangement numbers. As examples, we use the Abel-Zeilberger algorithm to prove the Paule-Schneider identities, an identity of Andrews and Paule, and an identity of Calkin.
\end{abstract}

\section{Introduction}

The main idea of this paper is to combine the classical lemma of Abel and Zeilberger's algorithm for hypergeometric sums to verify and to discover identities on non-hypergeometric sums. Abel's lemma [1] on summation by parts is stated as follows.

Lemma 1.1 For two arbitrary sequences $\left\{a_{k}\right\}$ and $\left\{b_{k}\right\}$, we have

$$
\sum_{k=m}^{n-1}\left(a_{k+1}-a_{k}\right) b_{k}=\sum_{k=m}^{n-1} a_{k+1}\left(b_{k}-b_{k+1}\right)+a_{n} b_{n}-a_{m} b_{m} .
$$

For a sequence $\left\{\tau_{k}\right\}$, define the forward difference operator $\Delta$ by

$$
\Delta \tau_{k}=\tau_{k+1}-\tau_{k} .
$$


Then Abel's lemma may be written as

$$
\sum_{k=m}^{n-1} b_{k} \Delta a_{k}=-\sum_{k=m}^{n-1} a_{k+1} \Delta b_{k}+a_{n} b_{n}-a_{m} b_{m}
$$

Graham, Knuth and Patashnik [12] reformulated Abel's lemma in terms of finite calculus to evaluate indefinite sums. Recently, Chu [8] utilized Able's lemma to prove basic hypergeometric identities including Bailey's very well poised ${ }_{6} \psi_{6}$-series identity by finding pairs $\left(a_{k}, b_{k}\right)$. Applying Abel's lemma to the pairs $\left(a_{k}, b_{k}\right)$, one obtains contiguous relations for the basic hypergeometric sums. Chen, Chen and $\mathrm{Gu}$ [5] presented a systematic approach to finding pairs $\left(a_{k}, b_{k}\right)$ by using the $q$-Gosper algorithm.

Let us illustrate how to combine Abel's lemma and Gosper's algorithm to prove identities involving harmonic numbers. The $k$-th harmonic number $H_{k}$ is given by

$$
H_{k}=\sum_{j=1}^{k} \frac{1}{j}
$$

Note that by definition, $H_{k}=0$ whenever $k \leq 0$. Let $f_{k}$ be a hypergeometric term, i.e., $f_{k+1} / f_{k}$ is a rational function of $k$. We consider the sum

$$
\sum_{k=m}^{n-1} f_{k} H_{k}
$$

We can use Gosper's algorithm [10] to determine whether there exists a hypergeometric term $a_{k}$ such that $\Delta a_{k}=f_{k}$. If such $a_{k}$ exists, by Abel's lemma we get

$$
\sum_{k=m}^{n-1} f_{k} H_{k}=\sum_{k=m}^{n-1} H_{k} \Delta a_{k}=-\sum_{k=m}^{n-1} \frac{a_{k+1}}{k+1}+a_{n} H_{n}-a_{m} H_{m} .
$$

Hence we can transform a sum involving harmonic numbers to a hypergeometric sum. We call the above procedure the Abel-Gosper algorithm.

The same idea applies to a definite sum

$$
S(n)=\sum_{k=0}^{n} F(n, k) H_{k}
$$

where $F(n, k)$ is a proper hypergeometric term such that $F(n, k)=0$ for $k>n$. In this case, we can apply Zeilberger's algorithm to find a hypergeometric term $G(n, k)$ and polynomials $p_{0}(n), \ldots, p_{d}(n)$ such that

$$
\sum_{j=0}^{d} p_{j}(n) F(n+j, k)=G(n, k+1)-G(n, k) .
$$


Taking

$$
f_{k}=\sum_{j=0}^{d} p_{j}(n) F(n+j, k)
$$

and

$$
a_{k}=G(n, k)
$$

in (1.4) and summing over $k$ from 0 to $n+d$, we deduce that

$$
\sum_{j=0}^{d} p_{j}(n) S(n+j)=-\sum_{k=0}^{n+d} \frac{G(n, k+1)}{k+1}+G(n, n+d+1) H_{n+d+1} .
$$

Moreover, we see that the sum on the right hand side of (1.5) is again a hypergeometric sum.

In order to apply the above approach to general holonomic sequences, we extend Abel's lemma by replacing the difference operator $\Delta$ with a linear operator $L$ of the form

$$
L a_{k}=r_{0}(k) a_{k}+r_{1}(k) a_{k+1}+\cdots+r_{d}(k) a_{k+d},
$$

where each $r_{i}(k)$ is a rational function of $k$. The extended version of Abel's lemma reads

$$
\sum_{k=m}^{n-1} b_{k} L^{*} a_{k}=\sum_{k=m}^{n-1} a_{k} L b_{k}-T(n)+T(m)
$$

where $L^{*}$ is the dual operator of $L$ defined by

$$
L^{*} a_{k}=r_{0}(k) a_{k}+r_{1}(k-1) a_{k-1}+\cdots+r_{d}(k-d) a_{k-d},
$$

and

$$
T(k)=\sum_{i=1}^{d} \sum_{j=1}^{i} r_{i}(k-j) a_{k-j} b_{k+i-j} .
$$

Let $f(n, k)$ be a hypergeometric term and let $g(n, k)$ be a function satisfying two recurrence relations

$$
g(n, k+d)=r_{1}(n, k) g(n, k)+\cdots+r_{d}(n, k) g(n, k+d-1)+u(n, k),
$$

and

$$
g(n+1, k)=s(n, k) g(n, k)+v(n, k),
$$

where the coefficients $r_{i}(n, k)$ and $s(n, k)$ are rational functions and $u(n, k), v(n, k)$ are hypergeometric terms. Notice that $g(n, k)$ is not necessarily a hypergeometric term. By iteration of (1.8), we deduce that

$$
g(n+i, k)=s_{i}(n, k) g(n, k)+v_{i}(n, k),
$$


where $s_{i}(n, k)$ is a rational function and $v_{i}(n, k)$ is a hypergeometric term. As in Zeilberger's algorithm, we aim to find $k$-free polynomials $p_{i}(n)$ and a hypergeometric term $a(n, k)$ such that

$$
\sum_{i=0}^{I} p_{i}(n) s_{i}(n, k) f(n+i, k)=L^{*} a(n, k),
$$

where $L^{*}$ is the linear operator given by

$$
L^{*} a(n, k)=-r_{1}(n, k) a(n, k)-\cdots-r_{d}(n, k-d+1) a(n, k+d-1)+a(n, k+d) .
$$

The polynomials $p_{i}(n)$ and the hypergeometric term $a(n, k)$ can be found by using Abramov's algorithm [2] or the convergence argument due to Chen, Paule and Saad [6]. Let

$$
S(n)=\sum_{k} f(n, k) g(n, k) .
$$

Applying the extended version of Abel's lemma (1.6), from (1.9) one can derive a recurrence relation for $S(n)$,

$$
\sum_{j=0}^{d} p_{j}(n) S(n+j)=\sum_{k} h(n, k)+T(n),
$$

where $p_{j}(n)$ are polynomials given in $(1.9), h(n, k)$ is a hypergeometric term. The above procedure for finding the recurrence relation (1.10) is called the Abel-Zeilberger algorithm.

We remark that (1.6) is equivalent to the statement

$$
b_{k} L^{*} a_{k}=a_{k} L b_{k}+\Delta(-T(k)),
$$

where $\Delta$ is the difference operator with respect to $k$. Using this relation, we may transform the sum $\sum_{k} b_{k} L^{*} a_{k}$ to the sum $\sum_{k} a_{k} L b_{k}$. Thus the Abel-Zeilberger algorithm can be viewed as a procedure to transform a non-hypergeometric sum to a hypergeometric sum. Notice that in the Abel-Zeilberger algorithm, $b_{k}$ is set to be $g(n, k)$ and $a_{k}=a(n, k)$ is determined by (1.9).

It should be mentioned that based on Karr's difference field theory [11], Schneider developed an algorithm for the following problem over recurrence terms in difference fields $[14,15]$. Given a summand $t_{k}$, we wish to find a summand $t_{k}^{\prime}$ and a function $g_{k}$ such that

$$
t_{k}=t_{k}^{\prime}+\Delta g_{k}
$$

and the sum $\sum_{k} t_{k}^{\prime}$ is easier to compute compared with $\sum_{k} t_{k}$. Observe that (1.11) can be formulated in the form of (1.12) by setting

$$
t_{k}=b_{k} L^{*} a_{k}, \quad t_{k}^{\prime}=a_{k} L b_{k}, \quad \text { and } \quad g_{k}=-T(k) .
$$

Therefore, we may use Schneider's algorithm instead of (1.11) to transform the sum $\sum_{k} b_{k} L^{*} a_{k}$ to the sum $\sum_{k} a_{k} L b_{k}$. However, it should be noted that Schneider's algorithm 
relies on the computation of $g_{k}$. In the Abel-Zeilberger algorithm, once $a_{k}$ and $b_{k}$ are found, then $t_{k}^{\prime}=a_{k} L b_{k}$ and $g_{k}$ is determined by $a_{k}$ and $b_{k}$. This means that in our case there is no need to compute $g_{k}$.

The paper is organized as follows. In Section 2, we give examples to demonstrate how to transform indefinite sums involving harmonic numbers to hypergeometric sums by using Abel-Gosper algorithm. Section 3 shows how to apply the Abel-Zeilberger algorithm to find recurrence relations of definite sums involving harmonic numbers and derangement numbers. This method applies to the Paule-Schneider identities, an identity due to Andrews and Paule, and an identity of Calkin.

\section{The Abel-Gosper algorithm}

In this section, we give examples to illustrate how to combine Abel's lemma and Gosper's algorithm to evaluate indefinite summations. We shall focus on sums involving harmonic numbers.

We begin with a simple example. Consider the sum

$$
S(n)=\sum_{k=1}^{n} H_{k}
$$

Expressing 1 as $\Delta k$, we obtain

$$
S(n)=\sum_{k=1}^{n} H_{k} \Delta k=-\sum_{k=1}^{n}(k+1) \Delta H_{k}+(n+1) H_{n+1}-H_{1}=(n+1) H_{n}-n .
$$

The following result is due to Spieß [16]. Here we give a derivation based on Abel's lemma.

Theorem 2.1 Let $H_{k}$ be the $k$-th harmonic number and $u(n)$ be a polynomial of degree $m$ in $n$. Then

$$
\sum_{k=0}^{n} u(k) H_{k}=p(n) H_{n}-q(n), \quad n=0,1,2, \ldots,
$$

where $p(n)$ and $q(n)$ are both polynomials of degree $m+1$ in $n$. Moreover, $p(n)$ is divisible by $n+1$.

Proof. It is well-known that there exists a polynomial $f(k)$ of degree $m+1$ in $k$ such that $\Delta f(k)=u(k)$ and the constant term of $f(k)$ is zero. Therefore, we may write $f(k)=k g(k)$, where $g(k)$ is a polynomial of degree $m$. From (1.4) it follows that

$$
\sum_{k=0}^{n} u(k) H_{k}=\sum_{k=0}^{n} \Delta(k g(k)) H_{k}=-\sum_{k=0}^{n} g(k+1)+(n+1) g(n+1) H_{n+1} .
$$


Since the sum $\sum_{k=0}^{n} g(k+1)$ is a polynomial of degree $m+1$ in $n$ and

$$
(n+1) g(n+1) H_{n+1}=(n+1) g(n+1) H_{n}+g(n+1),
$$

we arrive at (2.2).

Setting $u(n)=1$ in (2.2), we obtain (2.1). When $u(n)=n^{m}$ for $m=1,2,3$, we have

$$
\begin{aligned}
& \sum_{k=1}^{n} k H_{k}=\frac{n(n+1)}{2} H_{n}-\frac{(n-1) n}{4}, \\
& \sum_{k=1}^{n} k^{2} H_{k}=\frac{n(n+1)(2 n+1)}{6} H_{n}-\frac{(n-1) n(4 n+1)}{36}, \\
& \sum_{k=1}^{n} k^{3} H_{k}=\frac{n^{2}(n+1)^{2}}{4} H_{n}-\frac{(n-1) n(n+1)(3 n-2)}{48} .
\end{aligned}
$$

The same idea applies to the bonus problem 69 proposed by Graham, Knuth and Patashnik [12, Chapter 6].

Example 2.2 Find a closed form for

$$
\sum_{k=1}^{n} k^{2} H_{n+k}
$$

The above sum can be rewritten as

$$
\sum_{k=n+1}^{2 n}(k-n)^{2} H_{k}=\sum_{k=1}^{2 n}(k-n)^{2} H_{k}-\sum_{k=1}^{n}(k-n)^{2} H_{k}
$$

Expanding the summands and applying formulas (2.1), (2.3), (2.4), we obtain that

$$
\sum_{k=1}^{n} k^{2} H_{n+k}=\frac{n(n+1)(2 n+1)}{6}\left(2 H_{2 n}-H_{n}\right)-\frac{n(n+1)(10 n-1)}{36} .
$$

We remark that Chyzak [9] and Schneider [15] proved (2.7) by an extension of Zeilberger's algorithm and Karr's algorithm.

Example 2.3 Evaluate the sum

$$
\sum_{k=0}^{n-1} \frac{1}{4^{k}}\left(\begin{array}{c}
2 k \\
k
\end{array}\right) H_{k}
$$


By Gosper's algorithm, we find that

$$
\Delta \frac{2 k}{4^{k}}\left(\begin{array}{c}
2 k \\
k
\end{array}\right)=\frac{1}{4^{k}}\left(\begin{array}{c}
2 k \\
k
\end{array}\right) .
$$

Therefore,

$$
\begin{aligned}
\sum_{k=0}^{n-1} \frac{1}{4^{k}}\left(\begin{array}{c}
2 k \\
k
\end{array}\right) H_{k} & =-2 \sum_{k=0}^{n-1} \frac{1}{4^{k+1}}\left(\begin{array}{c}
2 k+2 \\
k+1
\end{array}\right)+\frac{2 n}{4^{n}}\left(\begin{array}{c}
2 n \\
n
\end{array}\right) H_{n} \\
& =2-\frac{n+1}{4^{n}}\left(\begin{array}{c}
2 n+2 \\
n+1
\end{array}\right)+\frac{2 n}{4^{n}}\left(\begin{array}{c}
2 n \\
n
\end{array}\right) H_{n} .
\end{aligned}
$$

Example 2.4 We have

$$
\sum_{k=0}^{n} H_{k}^{2}=(n+1) H_{n}^{2}-(2 n+1) H_{n}+2 n .
$$

Proof. Setting $a_{k}=k$ and $b_{k}=H_{k}^{2}$ in (1.1), we deduce that

$$
\begin{aligned}
\sum_{k=0}^{n} H_{k}^{2} & =-\sum_{k=0}^{n}(k+1) \Delta H_{k}^{2}+(n+1) H_{n+1}^{2} \\
& =-2 \sum_{k=0}^{n} H_{k}-\sum_{k=0}^{n} \frac{1}{k+1}+(n+1) H_{n+1}^{2} \\
& =-2(n+1) H_{n}+2 n-H_{n+1}+(n+1) H_{n+1}^{2} \\
& =(n+1) H_{n}^{2}-(2 n+1) H_{n}+2 n .
\end{aligned}
$$

Similarly, by setting $b_{k}=H_{k}^{3}$ in $(1.1)$, we deduce the following identities, see $[9,16]$,

$$
\begin{gathered}
\sum_{k=0}^{n} H_{k}^{3}=(n+1) H_{n}^{3}-\frac{3}{2}(2 n+1) H_{n}^{2}+3(2 n+1) H_{n}+\frac{1}{2} H_{n}^{(2)}-6 n, \\
\sum_{k=0}^{n}(2 k+1) H_{k}^{3}=(n+1)^{2} H_{n}^{3}-\frac{3}{2} n(n+1) H_{n}^{2}+\frac{3 n^{2}+3 n+1}{2} H_{n}-\frac{3}{4} n(n+1) .
\end{gathered}
$$

\section{The Abel-Zeilberger algorithm}

In this section, we use Abel's lemma and Zeilberger's algorithm to find recurrence relations for the definite sum of the form

$$
S(n)=\sum_{k=0}^{n} F(n, k) H_{k},
$$

where $F(n, k)$ is a hypergeometric term in $n$ and $k$. We shall give two examples. First, we consider an identity due to Chu and De Donno [7]. 
Example 3.1 For $n \geq 0$, we have

$$
\sum_{k=0}^{n}\left(\begin{array}{l}
n \\
k
\end{array}\right)^{2} H_{k}=\left(2 H_{n}-H_{2 n}\right)\left(\begin{array}{c}
2 n \\
n
\end{array}\right)
$$

Proof. Applying Zeilberger's algorithm to $\left(\begin{array}{l}n \\ k\end{array}\right)^{2}$, we obtain

$$
(n+1)\left(\begin{array}{c}
n+1 \\
k
\end{array}\right)^{2}-2(2 n+1)\left(\begin{array}{l}
n \\
k
\end{array}\right)^{2}=G(n, k+1)-G(n, k),
$$

where

$$
G(n, k)=(-3-3 n+2 k)\left(\begin{array}{c}
n \\
k-1
\end{array}\right)^{2}
$$

Let

$$
S(n)=\sum_{k=0}^{n}\left(\begin{array}{l}
n \\
k
\end{array}\right)^{2} H_{k}
$$

Substituting $F(n, k)=\left(\begin{array}{l}n \\ k\end{array}\right)^{2}$ and $G(n, k)=(-3-3 n+2 k)\left(\begin{array}{c}n \\ k-1\end{array}\right)^{2}$ into (1.5), we find that

$$
(n+1) S(n+1)-2(2 n+1) S(n)=\sum_{k=0}^{n+1} \frac{3 n-2 k+3}{k+1}\left(\begin{array}{l}
n \\
k
\end{array}\right)^{2}=\frac{4 n+1}{n+1}\left(\begin{array}{c}
2 n \\
n
\end{array}\right)
$$

where the second equality can be justified by applying Zeilberger's algorithm.

It is easy to verify that

$$
R(n)=\left(2 H_{n}-H_{2 n}\right)\left(\begin{array}{c}
2 n \\
n
\end{array}\right)
$$

satisfies the same recurrence relation (3.2). Since $S(0)=R(0)=0$, we get (3.1). This completes the proof.

Paule and Schneider [13] considered the following sums

$$
T_{n}^{(\alpha)}=\sum_{k=0}^{n}\left(1+\alpha(n-2 k) H_{k}\right)\left(\begin{array}{l}
n \\
k
\end{array}\right)^{\alpha}, \quad \alpha=1,2, \ldots
$$

They found closed forms of $T_{n}^{(\alpha)}$ for $1 \leq \alpha \leq 4$ and derived recurrence relations of $T_{n}^{(\alpha)}$ for $5 \leq \alpha \leq 9$. As will be seen, we can combine Abel's lemma and Zeilberger's algorithm to compute the sums $T_{n}^{(\alpha)}$. For example, let us consider the case $\alpha=3$.

Example 3.2 Let $T_{n}^{(3)}$ be defined by (3.3). For $n \geq 0$, we have

$$
T_{n}^{(3)}=(-1)^{n}
$$


Proof. Let

$$
F(n, k)=(n-2 k)\left(\begin{array}{l}
n \\
k
\end{array}\right)^{3} .
$$

By Zeilberger's algorithm, we find that

$$
F(n, k)+F(n+1, k)=G(n, k+1)-G(n, k),
$$

where

$$
G(n, k)=(2 n-k+2)\left(\begin{array}{c}
n \\
k-1
\end{array}\right)^{3}
$$

Let

$$
S(n)=T_{n}^{(3)}=\sum_{k=0}^{n}\left(1+3(n-2 k) H_{k}\right)\left(\begin{array}{l}
n \\
k
\end{array}\right)^{3} .
$$

By (1.5), we deduce that

$$
S(n)+S(n+1)=-3 \sum_{k=0}^{n+1} \frac{2 n-k+1}{k+1}\left(\begin{array}{l}
n \\
k
\end{array}\right)^{3}+\sum_{k=0}^{n}\left(\begin{array}{l}
n \\
k
\end{array}\right)^{3}+\sum_{k=0}^{n+1}\left(\begin{array}{c}
n+1 \\
k
\end{array}\right)^{3} .
$$

By Zeilberger's algorithm, we find that the right hand side, denoted by $R(n)$, satisfies

$$
(n+1) R(n)+(n+2) R(n+1)=0 .
$$

Since $R(0)=0$, we have $R(n)=0$ for $n=0,1, \ldots$ It is clear that $S(0)=1$. Thus we have $S(n)=(-1)^{n}$. This completes the proof.

Moreover, as a consequence of (1.5), we have the following property.

Theorem 3.3 Let $T_{n}^{(\alpha)}$ be given by (3.3) and

$$
U_{n}^{(\alpha)}=\sum_{k=0}^{n}(n-2 k)\left(\begin{array}{l}
n \\
k
\end{array}\right)^{\alpha} .
$$

Assume that the minimal recurrence relation for $U_{n}^{(\alpha)}$ computed by Zeilberger's algorithm is

$$
\sum_{i=0}^{d} p_{i}(n) U_{n+i}^{(\alpha)}=0 .
$$

Then the sum

$$
\sum_{i=0}^{d} p_{i}(n) T_{n+i}^{(\alpha)}
$$

is a hypergeometric sum. 
We find that the sum (3.4) equals zero for $\alpha=1,2, \ldots, 9$ and we conjecture that it holds for any nonnegative integer $\alpha$. We note that this conjecture implies the conjecture of Schneider and Paule [13], which says that $T_{n}^{(\alpha)}$ satisfies the minimal recurrence relation for $U_{n}^{(\alpha)}$ computed by Zeilberger's algorithm.

Notice that in the applications of Abel's lemma, the idea lies in the fact that $\Delta H_{k}$ is a hypergeometric term. In fact, there are other sequences satisfying similar properties that lead us to consider an extension of Abel's lemma.

Let $\left\{a_{k}\right\}$ be a sequence of numbers. We consider a linear operator $L$ of the form

$$
L a_{k}=r_{0}(k) a_{k}+r_{1}(k) a_{k+1}+\cdots+r_{d}(k) a_{k+d},
$$

where each $r_{j}(k)$ is a rational function of $k$. We associate the operator $L$ with a dual operator $L^{*}$ defined by

$$
L^{*} a_{k}=r_{0}(k) a_{k}+r_{1}(k-1) a_{k-1}+\cdots+r_{d}(k-d) a_{k-d} .
$$

In the above notation, Abel's lemma can be extended as follows.

Lemma 3.4 For two arbitrary sequences $\left\{a_{k}\right\}$ and $\left\{b_{k}\right\}$, we have

$$
\sum_{k=m}^{n-1} b_{k} L^{*} a_{k}=\sum_{k=m}^{n-1} a_{k} L b_{k}-T(n)+T(m),
$$

where

$$
T(k)=\sum_{i=1}^{d} \sum_{j=1}^{i} r_{i}(k-j) a_{k-j} b_{k+i-j} .
$$

Proof. It is easy to verify that

$$
\begin{aligned}
\sum_{k=m}^{n-1} b_{k} L^{*} a_{k} & =\sum_{k=m}^{n-1} \sum_{i=0}^{d} r_{i}(k-i) a_{k-i} b_{k} \\
& =\sum_{i=0}^{d} \sum_{k=m-i}^{n-1-i} r_{i}(k) a_{k} b_{k+i} \\
& =\sum_{k=m}^{n-1} r_{0}(k) a_{k} b_{k}+\sum_{i=1}^{d}\left[\sum_{k=m}^{n-1}+\sum_{k=m-i}^{m-1}-\sum_{k=n-i}^{n-1} r_{i}(k) a_{k} b_{k+i}\right. \\
& =\sum_{k=m}^{n-1} a_{k} L b_{k}+\sum_{i=1}^{d} \sum_{k=m-i}^{m-1} r_{i}(k) a_{k} b_{k+i}-\sum_{i=1}^{d} \sum_{k=n-i}^{n-1} r_{i}(k) a_{k} b_{k+i},
\end{aligned}
$$

as claimed. 
Let $f(n, k)$ be a bivariate hypergeometric term and $g(n, k)$ be a bivariate function. Assume that there exist rational functions $r_{j}(n, k)$ such that

$$
L g(n, k)=\sum_{j=0}^{d} r_{j}(n, k) g(n, k+j)
$$

is a bivariate hypergeometric term. In order to find a recurrence relation of order $I$ for the sum

$$
S(n)=\sum_{k=m}^{\ell} f(n, k) g(n, k),
$$

we proceed to find $k$-free polynomials $p_{i}(n)$, which are not all zeros, together with hypergeometric terms $a(n, k)$ and $w(n, k)$ such that

$$
\sum_{i=0}^{I} p_{i}(n) f(n+i, k) g(n+i, k)=g(n, k) L^{*} a(n, k)+w(n, k) .
$$

Summing (3.7) over $k$ and applying the extended version of Abel's lemma, we deduce that

$$
\begin{aligned}
\sum_{i=0}^{I} p_{i}(n) S(n+i) & =\sum_{i=0}^{I} \sum_{k=m}^{\ell} p_{i}(n) f(n+i, k) g(n+i, k) \\
& =\sum_{k=m}^{\ell}\left(g(n, k) L^{*} a(n, k)+w(n, k)\right) \\
& =\sum_{k=m}^{\ell} a(n, k) L g(n, k)+\sum_{k=m}^{\ell} w(n, k)-T(\ell+1)+T(m),
\end{aligned}
$$

where $T(k)$ is given by (3.6). Notice that in the last expression, the two summands are hypergeometric.

To solve equation (3.7), we shall restrict our attention to the functions $g(n, k)$ satisfying the condition

$$
g(n+1, k)=s(n, k) g(n, k)+v(n, k),
$$

where $s(n, k)$ is a rational function and $v(n, k)$ is a hypergeometric term. By induction, it is easy to show that there exist rational functions $s_{i}(n, k)$ and hypergeometric terms $v_{i}(n, k)$ such that

$$
g(n+i, k)=s_{i}(n, k) g(n, k)+v_{i}(n, k) .
$$

Now we can solve the following equation for $p_{i}(n)$ and $a(n, k)$ by a variation of Zeilberger's algorithm

$$
\sum_{i=0}^{I} p_{i}(n) f(n+i, k) s_{i}(n, k)=L^{*} a(n, k) .
$$


It can be checked that $a(n, k)$ is similar to $f(n, k)$, that is,

$$
R(n, k)=\frac{a(n, k)}{f(n, k)}
$$

is a rational function of $n$ and $k$. Hence (3.10) is equivalent to

$$
\sum_{i=0}^{I} p_{i}(n) \frac{f(n+i, k)}{f(n, k)} s_{i}(n, k)=\sum_{j=0}^{d} \frac{f(n, k-j)}{f(n, k)} r_{j}(n, k-j) R(n, k-j) .
$$

Since $f(n, k)$ is hypergeometric, both $f(n+i, k) / f(n, k)$ and $f(n, k-j) / f(n, k)$ are rational functions. Therefore, (3.11) is a non-homogenous linear recurrence equation on $R(n, k)$ with parameters $p_{i}(n)$, which can be solved by using Abramov's algorithm [2] or by the convergence argument due to Chen, Paule and Saad [6].

Once we find a solution $\left(p_{0}(n), \ldots, p_{I}(n), a(n, k)\right)$ of equation $(3.10)$, it is easy to check that

$$
\left(p_{0}(n), \ldots, p_{I}(n), a(n, k), w(n, k)\right)
$$

is a solution of equation (3.7), where

$$
w(n, k)=\sum_{i=0}^{I} p_{i}(n) f(n+i, k) v_{i}(n, k) .
$$

It should be noted that we do not have an upper bound on $I$. So it is not guaranteed that the algorithm will succeed in finding a recurrence relation. In practice, we only need to consider small values of $I$. In summary, the Abel-Zeilberger algorithm can be described as follows.

Input: The order $I$ of the recurrence relation, a hypergeometric term $f(n, k)$ and a function $g(n, k)$ satisfying two recurrence relations

$$
g(n, k+d)=r_{1}(n, k) g(n, k)+\cdots+r_{d}(n, k) g(n, k+d-1)+u(n, k),
$$

and

$$
g(n+1, k)=s(n, k) g(n, k)+v(n, k),
$$

where $r_{i}(n, k)$ and $s(n, k)$ are rational functions, and $u(n, k)$ and $v(n, k)$ are hypergeometric terms.

Output: $k$-free polynomials $p_{i}(n)(0 \leq i \leq I)$, hypergeometric terms $t_{1}(n, k), t_{2}(n, k)$ and a function $T(k)$ satisfying

$$
\sum_{i=0}^{I} p_{i}(n) S(n+i)=\sum_{k=m}^{\ell} t_{1}(n, k)+\sum_{k=m}^{\ell} t_{2}(n, k)-T(\ell+1)+T(m),
$$

where

$$
S(n)=\sum_{k=m}^{\ell} f(n, k) g(n, k)
$$


The algorithm consists of the following steps.

Step 1. For $0 \leq i \leq I$, compute the rational functions $s_{i}(n, k)$ and the hypergeometric terms $v_{i}(n, k)$ defined by (3.9) based on the recurrence relations

$$
\begin{aligned}
& s_{i+1}(n, k)=s(n+i, k) s_{i}(n, k), \\
& v_{i+1}(n, k)=s(n+i, k) v_{i}(n, k)+v(n+i, k),
\end{aligned}
$$

with the initial values $s_{0}(n, k)=1$ and $v_{0}(n, k)=0$.

Step 2. Let

$$
L g(n, k)=-r_{1}(n, k) g(n, k)-\cdots-r_{d}(n, k) g(n, k+d-1)+g(n, k+d) .
$$

According to (3.11), establish an equation on $p_{i}(n)$ and $R(n, k)=a(n, k) / f(n, k)$. That is, set

$$
P_{i}(n, k)=\frac{f(n+i, k)}{f(n, k)} s_{i}(n, k) \quad \text { and } \quad Q_{j}(n, k)=\frac{f(n, k-j)}{f(n, k)} r_{j}(n, k-j),
$$

so that (3.11) takes the form

$$
\sum_{i=0}^{I} p_{i}(n) P_{i}(n, k)=\sum_{j=0}^{d} Q_{j}(n, k) R(n, k-j) .
$$

Step 3. Solve equation (3.16) for $R(n, k)$ and $p_{i}(n)(0 \leq i \leq I)$ by using Abramov's algorithm.

Step 4. Compute $w(n, k)$ based on (3.12). For $L$ given by (3.15), compute $T(k)$ according to (3.6). Finally, set

$$
t_{1}(n, k)=a(n, k) u(n, k) \quad \text { and } \quad t_{2}(n, k)=w(n, k) .
$$

Then $\left(p_{i}(n), t_{1}(n, k), t_{2}(n, k), T(k)\right)$ is the desired output.

Here we give two examples. The first involves the $n$-th derangement number $D_{n}$ as given by

$$
D_{n}=n ! \sum_{k=0}^{n} \frac{(-1)^{k}}{k !}
$$

Using MacMahon's partition analysis, Andrews and Paule [3] obtained the following identity. We shall give a derivation by applying the Abel-Zeilberger algorithm.

Example 3.5 For $n \geq N \geq n-k$, we have

$$
\sum_{j \geq 0}\left(\begin{array}{l}
k \\
j
\end{array}\right) \frac{(k+n-j) !}{(k+N-j) !} D_{k+N-j}=(-1)^{n} \sum_{j \geq 0}(-1)^{j}\left(\begin{array}{l}
n \\
j
\end{array}\right)(j+k) !
$$


Proof. Substituting $k+N-j$ for $j$, the left hand side of (3.17) can be rewritten as

$$
S(N, n)=\sum_{j=N}^{k+N}\left(\begin{array}{c}
k \\
j-N
\end{array}\right) \frac{(n+j-N) !}{j !} D_{j}
$$

Because of the recurrence relation

$$
D_{n}=n D_{n-1}+(-1)^{n}
$$

we may take

$$
f(N, j)=\left(\begin{array}{c}
k \\
j-N
\end{array}\right) \frac{(n+j-N) !}{j !} \text { and } g(N, j)=D_{j}
$$

as the input of the Abel-Zeilberger algorithm. Then we obtain

$$
S(N, n)-S(N+1, n)=\sum_{j \geq N}(-1)^{j}\left(\begin{array}{c}
k \\
j-N
\end{array}\right) \frac{(n+j-N) !}{(j+1) !} .
$$

Denote the right hand side by $G(k)$. By Zeilberger's algorithm, we find that for $k \geq 0$,

$$
(1+N-n+k) G(k)-(2+k+N) G(k+1)=0,
$$

which implies that $G(k)=0$ for $k \geq n-N \geq 0$. Thus we get $S(N, n)=S(N+1, n)$. In particular,

$$
S(N, n)=S(n, n)=\sum_{j \geq n}\left(\begin{array}{c}
k \\
j-n
\end{array}\right) D_{j}
$$

Applying the Abel-Zeilberger algorithm to

$$
f(n, j)=\left(\begin{array}{c}
k \\
j-n
\end{array}\right) \text { and } g(n, j)=D_{j}
$$

we find

$$
(n+1) S(n, n)+(n+k+1) S(n+1, n+1)-S(n+2, n+2)=\sum_{j \geq n}(-1)^{j}\left(\begin{array}{l}
k+1 \\
j-n
\end{array}\right)=0 .
$$

By Zeilberger's algorithm, we find that the right hand side of (3.17) satisfies the same recurrence relation. Finally, from the identity

$$
\sum_{j=0}^{k}\left(\begin{array}{l}
k \\
j
\end{array}\right) D_{j}=k !
$$

we deduce that $S(0,0)=k$ ! and $S(1,1)=(k+1) !-k$, which coincides with the initial values of the right hand side of (3.17). Thus (3.17) holds for $n \geq N \geq n-k$. 
We remark that in the above proof we obtain a recurrence relation of order 1 for the sum $S(N, n)$, that is, $S(N, n)=S(N+1, n)$, and the creative telescoping for $\Pi \Sigma$-fields gives a recurrence relation of order 2 .

To conclude this paper, we consider the following sums,

$$
S_{n}^{(\alpha)}=\sum_{k=0}^{n}\left(\sum_{j=0}^{k}\left(\begin{array}{l}
n \\
j
\end{array}\right)\right)^{\alpha}
$$

For $\alpha=1,2$ and 3, closed forms for $S_{n}^{(\alpha)}$ have been derived by Andrews and Paule [3] by using the method of MacMahon's partition analysis. Indeed, these formulas can be derived by using the Abel-Zeilberger algorithm.

Example 3.6 We have

$$
S_{n}^{(1)}=\sum_{k=0}^{n} \sum_{j=0}^{k}\left(\begin{array}{l}
n \\
j
\end{array}\right)=n 2^{n-1}+2^{n}
$$

and

$$
S_{n}^{(2)}=\sum_{k=0}^{n}\left(\sum_{j=0}^{k}\left(\begin{array}{l}
n \\
j
\end{array}\right)\right)^{2}=\left(\frac{n}{2}+1\right) 2^{2 n}-\frac{n}{2}\left(\begin{array}{c}
2 n \\
n
\end{array}\right) .
$$

Proof. Let

$$
f(n, k)=1, \quad g(n, k)=\sum_{j=0}^{k}\left(\begin{array}{l}
n \\
j
\end{array}\right)
$$

It is clear that

$$
g(n, k+1)=g(n, k)+\left(\begin{array}{c}
n \\
k+1
\end{array}\right) \quad \text { and } g(n+1, k)=2 g(n, k)-\left(\begin{array}{l}
n \\
k
\end{array}\right) .
$$

Applying the Abel-Zeilberger algorithm to $f(n, k)$ and $g(n, k)$, we find that

$$
S_{n}^{(1)}=\sum_{k=0}^{n}(C-k)\left(\begin{array}{c}
n \\
k+1
\end{array}\right)-T(n+1)+T(0),
$$

where $T(k)=(C+1-k) g(n, k)$ and $C$ is a constant. Setting $C=-1$, we get $T(0)=0$. Thus

$$
\begin{aligned}
S_{n}^{(1)} & =-\sum_{k=0}^{n}(k+1)\left(\begin{array}{c}
n \\
k+1
\end{array}\right)+(n+1) g(n, n+1) \\
& =-n \sum_{k=0}^{n}\left(\begin{array}{c}
n-1 \\
k
\end{array}\right)+(n+1) \sum_{j=0}^{n+1}\left(\begin{array}{l}
n \\
j
\end{array}\right) \\
& =-n 2^{n-1}+(n+1) 2^{n} \\
& =n 2^{n-1}+2^{n} .
\end{aligned}
$$


Now we consider the evaluation of $S_{n}^{(2)}$. Let $f(n, k), g(n, k)$ be given as above, and let $h(n, k)=g(n, k)^{2}$. From (3.21), we see that

$$
\begin{aligned}
& h(n, k+1)=h(n, k)+u(n, k), \\
& h(n+1, k)=4 h(n, k)+v(n, k),
\end{aligned}
$$

where

$$
\begin{aligned}
& u(n, k)=2\left(\begin{array}{c}
n \\
k+1
\end{array}\right) g(n, k)+\left(\begin{array}{c}
n \\
k+1
\end{array}\right)^{2}, \\
& v(n, k)=-4\left(\begin{array}{l}
n \\
k
\end{array}\right) g(n, k)+\left(\begin{array}{l}
n \\
k
\end{array}\right)^{2} .
\end{aligned}
$$

It should be mentioned that there is no need to impose the condition for $u(n, k)$ and $v(n, k)$ to be hypergeometric in the Abel-Zeilberger algorithm. This means that we can apply the Abel-Zeilberger algorithm to $f(n, k)$ and $h(n, k)$ to deduce that

$S_{n}^{(2)}=\sum_{k=0}^{n}(C-k)\left(2\left(\begin{array}{c}n \\ k+1\end{array}\right) g(n, k)+\left(\begin{array}{c}n \\ k+1\end{array}\right)^{2}\right)+(n-C) h(n, n+1)+(C+1) h(n, 0)$,

where $C$ is a constant. Setting $C=n / 2-1$ and applying the Abel-Zeilberger algorithm, we find that

$$
\sum_{k=0}^{n}(n / 2-1-k)\left(\begin{array}{c}
n \\
k+1
\end{array}\right) g(n, k)=\sum_{k=0}^{n} \frac{-n+k+1}{2}\left(\begin{array}{c}
n \\
k+1
\end{array}\right)^{2}-\frac{n}{2} g(n, 0) .
$$

Hence

$$
\begin{aligned}
& S_{n}^{(2)}=\sum_{k=0}^{n}(-n+k+1)\left(\begin{array}{c}
n \\
k+1
\end{array}\right)^{2}-n+\sum_{k=0}^{n}\left(\frac{n}{2}-1-k\right)\left(\begin{array}{c}
n \\
k+1
\end{array}\right)^{2}+\left(\frac{n}{2}+1\right) 2^{2 n}+\frac{n}{2} \\
& =-\frac{n}{2} \sum_{k=0}^{n}\left(\begin{array}{c}
n \\
k+1
\end{array}\right)^{2}+\left(\frac{n}{2}+1\right) 2^{2 n}-\frac{n}{2} \\
& =-\frac{n}{2}\left(\left(\begin{array}{c}
2 n \\
n
\end{array}\right)-1\right)+\left(\frac{n}{2}+1\right) 2^{2 n}-\frac{n}{2} \\
& =\left(\frac{n}{2}+1\right) 2^{2 n}-\frac{n}{2}\left(\begin{array}{c}
2 n \\
n
\end{array}\right) .
\end{aligned}
$$

Using the same argument, one can derive Calkin's identity [4]

$$
S_{n}^{(3)}=n 2^{3 n-1}+2^{3 n}-3 n 2^{n-2}\left(\begin{array}{c}
2 n \\
n
\end{array}\right) .
$$

Acknowledgments. We wish to thank Professor Lu Yang and the referee for valuable comments. This work was supported by the 973 Project, the PCSIRT Project of the Ministry of Education, and the National Science Foundation of China. 


\section{References}

[1] N.H. Abel, Untersuchungen über die Reihe $1+\frac{m}{1} x+\frac{m(m-1)}{1 \cdot 2} x^{2}+\cdots$, J. Reine Angew. Math. 1 (1826) 311-339.

[2] S.A. Abramov, Rational solutions of linear difference and $q$-difference equations with polynomial coefficients, Programmirovanie 6 (1999) 74-80.

[3] G.E. Andrews and P. Paule, MacMahon' s partition analysis IV: Hypergeometric multisums, Śem. Lothar. Combin. 42 (1999) 1-24.

[4] N.J. Calkin, A curious binomial identity, Discrete Math. 131 (1994) 335-337.

[5] V.Y.B. Chen, W.Y.C. Chen, and N.S.S. Gu, The Abel Lemma and the $q$-Gosper algorithm, Math. Comput. 77 (2008) 1057-1074.

[6] W.Y.C. Chen, P. Paule, and H. Saad, Converging to Gosper's algorithm, Adv. Appl. Math. 41 (2008) 351-364.

[7] W.C. Chu and L. De Donno, Hypergeometric series and harmonic number identities, Adv. Appl. Math. 34 (2005) 123-137.

[8] W.C. Chu, Abel's lemma on summation by parts and basic hypergeomietric series, Adv. Appl. Math. 39 (2007) 490-514.

[9] F. Chyzak, An extension of Zeilberger's fast algorithm to general holonomic functions, Discrete Math. 217 (2000) 115-134.

[10] R.W. Gosper, Decision procedures for indefinite hypergeometric summation, Proc. Nat. Acad. Sci. U.S.A. 75 (1978) 40-42.

[11] M. Karr, Summation in finite terms, J. ACM 28 (1981) 305-350.

[12] R.L. Graham, D.E. Knuth and O. Patashnik, Concrete Mathematics: a Foundation for Computer Science, Addison-Wesley Publishing Company, Amsterdam, 2nd edition, 1994.

[13] P. Paule and C. Schneider, Computer proofs of a new family of harmonic number identities, Adv. Appl. Math. 31 (2003) 359-378.

[14] C. Schneider, A new Sigma approach to multi-summation, Adv. Appl. Math. 34 (2005) 740-767.

[15] C. Schneider, Symbolic summation assists combinatorics, Sém. Lothar. Combin. 56 (2006) Article B56b.

[16] J. Spieß, Some identities involving harmonic numbers, Math. Comput. 55 (1990) 839-863. 